\title{
Lessons from BWS twins: complex maternal and paternal hypomethylation and a common source of haematopoietic stem cells
}

Jet Bliek $^{*}{ }^{1}$, Marielle Alders ${ }^{1}$, Saskia M Maas ${ }^{1,2}$, Roelof-Jan Oostra ${ }^{3}$, Deborah M Mackay ${ }^{4,5}$, Karin van der Lip ${ }^{1}$, Johnatan L Callaway ${ }^{4,5}$, Alice Brooks ${ }^{6}$, Sandra van 't Padje ${ }^{1}$, Andries Westerveld ${ }^{7}$, Nico J Leschot ${ }^{1}$ and Marcel MAM Mannens ${ }^{1}$

\author{
${ }^{1}$ Department of Clinical Genetics, Academic Medical Centre, Amsterdam, The Netherlands; ${ }^{2}$ Department of \\ Paediatrics, Academic Medical Centre, Amsterdam, The Netherlands; ${ }^{3}$ Department of Anatomy and Embryology, \\ Academic Medical Centre, Amsterdam, The Netherlands; ${ }^{4}$ Division of Human Genetics University of Southampton, \\ Southampton, UK; ${ }^{5}$ Wessex Regional Genetics Laboratory, Salisbury District Hospital, Salisbury, UK; ${ }^{6}$ Department of \\ Clinical Genetics, Erasmus University, Rotterdam, The Netherlands; ${ }^{7}$ Department of Human Genetics, Academic \\ Medical Centre, Amsterdam, The Netherlands
}

The Beckwith-Wiedemann syndrome (BWS) is a growth disorder for which an increased frequency of monozygotic (MZ) twinning has been reported. With few exceptions, these twins are discordant for BWS and for females. Here, we describe the molecular and phenotypic analysis of 12 BWS twins and a triplet; seven twins are $M Z$, monochorionic and diamniotic, three twins are $M Z$, dichorionic and diamniotic and three twins are dizygotic. Twelve twins are female. In the majority of the twin pairs (11 of 13), the defect on chromosome 11p15 was hypomethylation of the paternal allele of DMR2. In 5 of 10 twins, there was additional hypomethylation of imprinted loci; in most cases, the loci affected were maternally methylated, but in two cases, hypomethylation of the paternally methylated DLK1 and H19 DMRs was detected, a novel finding in BWS. In buccal swabs of the MZ twins who share a placenta, the defect was present only in the affected twin; comparable hypomethylation in lymphocytes was detected in both the twins. The level of hypomethylation reached levels below $25 \%$. The exchange of blood cells through vascular connections cannot fully explain the degree of hypomethylation found in the blood cell of the non-affected twin. We propose an additional mechanism through which sharing of aberrant methylation patterns in discordant twins, limited to blood cells, might occur. In a BWS-discordant MZ triplet, an intermediate level of demethylation was found in one of the non-affected sibs; this child showed mild signs of BWS. This finding supports the theory that a methylation error proceeds and possibly triggers the twinning process.

European Journal of Human Genetics (2009) 17, 1625-1634; doi:10.1038/ejhg.2009.77; published online 10 June 2009

Keywords: twinning; Beckwith-Wiedemann syndrome; methylation defect; chromosome 11p15; hypomethylation of imprinted loci; vascular connections

\footnotetext{
*Correspondence: Dr J Bliek, Department of Clinical Genetics, DNA diagnostics, Academic Medical Centre, Meibergdreef 15, Amsterdam $1105 \mathrm{AZ}$, The Netherlands.

Tel: + 312056678 99; Fax: + 312056693 89;

E-mail: j.bliek@amc.uva.nl

Received 18 December 2008; revised 2 April 2009; accepted 7 April 2009; published online 10 June 2009
}

Beckwith and Wiedemann ${ }^{1,2}$ reported a syndrome of macroglossia and omphalocele, associated with adrenal cortical cytomegaly, fetal gigantism and other abnormalities. The Beckwith-Wiedemann syndrome (BWS) is thus characterized by overgrowth during fetal development and, as subsequently documented, a 
markedly increased susceptibility to certain childhood tumours. $^{3-6}$

Two imprinted gene clusters on chromosome $11 \mathrm{p} 15.5$ are involved in the aetiology of BWS. The more telomeric cluster (BWSIC1) contains the embryonal growth factor IGF2 and a non-coding RNA, H19, expressed from the paternally and maternally derived alleles, respectively. Parentally restricted expression of the genes in this cluster is regulated through an imprinting centre (IC1) that contains a differentially methylated region, DMR1 (DMRH19), located within the promoter of H19. The more centromeric cluster, under the control of imprinting centre 2 (BWSIC2), contains the cell cycle inhibitor CDKN1C and an antisense RNA, KCNQ1OT1. Expression of these genes is regulated by a maternally methylated imprinting centre (IC2) containing a DMR (DMR2 or KvDMR) located within KCNQ1 (see Figure 1).

Among BWS patients, different genetic and epigenetic lesions of $11 \mathrm{p} 15$ have been detected. In the majority of patients, an imprinting defect is present: 55\% of patients manifest loss of maternal methylation of DMR2, and $5 \%$ have gain of methylation of DMR1 on the maternal chromosome. In $20 \%$ of the patients, genetic defects of 11 p15 such as uniparental disomy, translocations and trisomies have been detected, and $5 \%$ of the patients have loss of function mutations in $C D K N 1 C$; in the remaining patients, the cause of disease remains unknown. ${ }^{7-10}$

In a subset of BWS patients (20\%) with an imprinting defect of DMR2, an additional loss of methylation has been detected at other imprinted loci throughout the genome, including PLAGL1, NESPAS, PEG3, IGF2R, SNRPN and
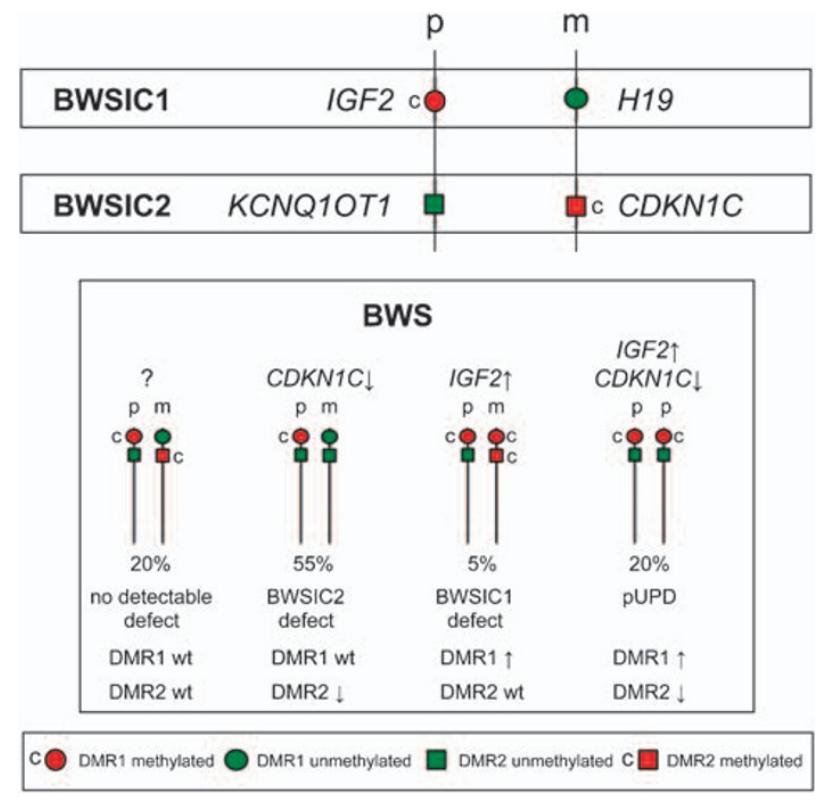

Figure 1 Schematic representation of the two imprinting clusters on chromosome $11 \mathrm{p} 15$ and their imprinting defects found among BWS patients. p: paternal allele, m: maternal allele, c: methylated.
GRB10. ${ }^{11,12}$ Hypomethylation of multiple maternally methylated imprinted loci, including KCNQ1OT1, was also described in patients with transient neonatal diabetes. ${ }^{13}$

Phenotypic discordance between monozygotic (MZ) ('identical') twins is a well recognized though incompletely understood phenomenon. MZ twins occur in the population at a rate of $3-4$ per 1000 births and at a sex ratio approximating 1:1, although female twinning is more prevalent in later twinning events. ${ }^{14} \mathrm{MZ}$ twins derive from a single zygote and therefore should have, in theory, identical genomes, apart from somatically acquired (epi)mutations. However, many examples of genetic differences between MZ twins have been reported, ${ }^{15-18}$ indicating that these somatic changes may occur soon after conception. MZ twinning occurs in a time frame spanning 4-9 days after conception, and the developmental stage of twinning is indicated by the resultant placentation of the twins.

Twenty-five percent/one-third of $\mathrm{MZ}$ twins have separate placentas; dichorionic MZ (DC-MZ) twinning is postulated to occur 0-4 days after fertilisation, before the formation of the blastocyst. Monochorionic monzygotic (MC-MZ) twins result from twinning events approximately $>4$ days after fertilisation, after the chorion is formed and therefore share a placenta. The majority of MC-MZ twins have separate amniotic membranes (diamniotic); in the rare cases where twinning occurs relatively late $(<5 \%)$, the twins also share a single amniotic sac.

By contrast, with MZ twins, dizygotic (DZ) twinning occurs when two oocytes mature and become fertilised by two different sperm cells at the same time. DZ twins have equivalent genetic concordance to siblings. The frequency of dizygotic twinning is about 9-20 per 1000 live births in Europe and the sex ratio approximates $1: 1{ }^{19} \mathrm{DZ}$ twins have separate placentas and amniotic membranes, although occasionally the placentas can be fused and vascular connections may be present. Several monochorionic dizygotic twins have been reported, almost exclusively cases of assisted reproduction (ART). ${ }^{20}$

In the literature, a large number of BWS twins have been described. Remarkably, the great majority of BWS twins are $\mathrm{MZ}$, but they are discordant, that is, one twin presents with BWS, whereas the other is unaffected (though an unaffected twin may have some features of the disease). Moreover, the majority of BWS twins show hypomethylation of DMR2. There is a striking excess of female twins, suggesting a possible mechanistic link with $\mathrm{X}$-chromosome inactivation. ${ }^{21}$

Machin $^{22}$ hypothesized that discordancy of XI in MZ twinning may result from mutual repulsion of cell clones with different XI. Bestor ${ }^{23}$ further proposed that the failure of DNA methylation maintenance in a single cell in early zygotic development could introduce epigenetic and hence functional asymmetry into the zygote, and potentially precipitate a twinning event. The molecular basis of this failure is thought to be a Dmnt1o defect, occurring at an 
8-cell stage. Bestor ${ }^{23}$ suggested that complete discordance, with no normally imprinted cells in one twin and no aberrantly imprinted cells in the other, would arise from a twinning event close to the time of the imprinting error, whereas temporal dissociation of these events might lead to partial discordancy where the affected twin was mosaic for normal and aberrantly imprinted cells.

In a cohort of 250 BWS patients described by Weksberg, ${ }^{24}$ the frequency of $\mathrm{MZ}$ twinning was $8 \%$ as compared with $1 \% \mathrm{DZ}$ twinning; in all MZ twins (10 of 10), there was phenotypic discordance and hypomethylation of DMR2 was the cause of disease, and 8 of 10 pairs were female. In a cohort of 79 patients, Gaston ${ }^{27}$ identified five multiple births: two sets of MZ female twins, two female DZ twin pairs and one DZ twin pair of mixed sex. The two MZ twin pairs both showed hypomethylation at DMR2. It was postulated that DMR2 hypomethylation resulted from a failure of methylation maintenance either coincident with or shortly after the twinning event. ${ }^{24}$

Other genetic defects have been found in BWS twins: ${ }^{25}$ uniparental disomy in a male $\mathrm{MZ}$ twin pair ${ }^{26}$ and a mosaic trisomy $11 \mathrm{p} 15$ in a female $\mathrm{MZ}$ twin pair. ${ }^{27}$

In the majority of BWS cases, molecular diagnosis relies on analysis of leukocyte-derived DNA. In two large studies, ${ }^{24,28}$ DMR2 hypomethylation was found in DNA derived from blood samples of both the affected and nonaffected twins of discordant twin pairs. It has been proposed that aberrant methylation in the blood of the healthy twin is caused by vascular connections in the placenta that is shared by both MZ (monochorionic, diamnionic) twins. ${ }^{24}$

In this study, we describe the epigenetic and phenotypic analysis of 13 discordant BWS twin pairs, including DZ, DC-MZ and MC-MC pairs. We determined the nature and frequency of their epigenetic defects at 11 p15 and at other imprinted loci, and we investigated whether vascular connections could account for the epigenetic anomalies seen in healthy twins of discordant BWS.

\section{Materials and methods}

Most twins were referred to our laboratory for routine diagnostic screening for BWS. Twin 7 is described by ${ }^{27}$ and twin 8 was referred to us by Dr LM Yates, Newcastle on Tyne, UK, for research purposes. DNA was extracted from blood lymphocytes, buccal swabs and/or saliva.

Clinical data were obtained by sending questionnaires to referring paediatricians. Data on placentation were obtained, when available, from the original pathological report of the placenta. Birthweight centiles were calculated according to Gielen et al. ${ }^{29}$

Zygosity was established with the use of PowerPlex 16 (Promega Corporation, Madison, WI, USA). Zygosity testing was performed on DNA isolated from buccal swabs when available; in all other cases, it was performed on DNA isolated from blood lymphocytes. Methylation analysis of DMR1 and DMR2 were performed either by southern blot analysis, as described earlier, ${ }^{9}$ or by HRMA as described by Alders et $a l^{30}$ and MS-PCR as described earlier. ${ }^{13}$ Analysis of loci other then chromosome 11p15 was performed by MS-PCR.

$\mathrm{X}$-chromosome inactivation was performed as described by Allen et $a l^{31}$ and calculated according to Horsthemke et al. ${ }^{32}$

\section{Results \\ Zygosity, placentation and female-to-male ratio} The findings concerning zygosity, placentation and femaleto-male ratios are summarised in Table 1.

The rate of $\mathrm{MZ}$ twinning is increased among BWS patients. Our diagnostic laboratory received 13 BWS twin pairs (12 twins and one triplet) among 400 BWS patients referred for routine diagnostic screening. The dizygotic twinning rate was normal compared with the normal population (0.75 (3 of 400) vs $0.70-1.1 \%$ ). The MZ twinning rate was increased, with a frequency of $2.5 \%$ (10 of 400 ) as compared with $0.3-0.4 \%$ among normal twins. ${ }^{24,33}$ The distribution of monochorionic and dichorionic twins among MZ twins was similar to that in the normal population. Placentation was known in two out of the three dizygotic twins in this study. They were both conceived by ART $(1 \times$ ovulation induction, $1 \times$ IVF). Both cases showed separate placentas.

All the three DZ twins were female, and 9 of $10 \mathrm{MZ}$ twins were female. Although the numbers are small, this differs from what is observed among normal twins where there is a very small shift towards females $\left(0.496-0.512^{33}\right)$ and is consistent with the findings of Weksberg et al. ${ }^{24}$

\section{(Epi) genotype}

The methylation profiles of all twins are summarised in Table 2.

In 11 of 13 cases, the (epi) genetic defect was hypomethylation of the maternal DMR2 (KCNQ1OT1). In one case (twin 7), a mosaic trisomy of chromosome $11 \mathrm{p}$ was found to result in an increased methylation level of DMR1 (H19) and a decreased methylation level of DMR2 (KCNQ1OT1). In one case (twin 12), there was no detectable genetic defect. A CDKN1C mutation was excluded in these twins.

In six of the seven $M Z$ twins who shared a placenta (MZ MC-DA), demethylation of DMR2 could be detected in the blood of both the affected and the non-affected twin. In buccal swabs, the defect was present only in the twin with the BWS phenotype in five twins (Figure 2a). A different situation is present in the triplet $3 \mathrm{~A}-3 \mathrm{C}$ (Figure 2b). The affected sib (3A) showed a similar degree 
Table 1 Zygosity, placentation and female-to-male ratio

\begin{tabular}{lcc}
\hline Zygosity & $\begin{array}{c}\text { Dizygotic } \\
\text { (DZ) }\end{array}$ & $\begin{array}{c}\text { Monozygotic } \\
\text { (MZ) }\end{array}$ \\
\hline BWS twins in this study & 3 & $10^{\mathrm{a}}$ \\
Percentage of BWS patients (400) & $0.75 \%$ & $2.5 \%$ \\
Population prevalence & $0.7-1.1 \%$ & $0.3-0.4 \%$
\end{tabular}

\begin{tabular}{lccc} 
& \multicolumn{2}{c}{ Placentation } \\
& $\begin{array}{c}\text { Dizygotic } \\
(D Z)\end{array}$ & $\begin{array}{c}\text { Monochromic } \\
\text { diamniotic } \\
(\text { MC-DA) }\end{array}$ & $\begin{array}{c}\text { Dichorionic } \\
\text { diamniotic } \\
(D C-D A)\end{array}$ \\
\hline $\begin{array}{l}\text { BWS twins in this } \\
\text { study }\end{array}$ & $3^{\mathrm{b}}$ & $7^{\mathrm{a}}$ & 3 \\
$\begin{array}{l}\text { Percentage of MZ } \\
\text { twins (10) }\end{array}$ & - & $70 \%$ & $30 \%$ \\
$\begin{array}{l}\text { Percentage among } \\
\text { nomal twins }\end{array}$ & - & $66.6 \%$ & $33.3 \%$
\end{tabular}

normal twins

\begin{tabular}{|c|c|c|}
\hline \multicolumn{3}{|c|}{ Female-to-male ratio (Female/Male) } \\
\hline & $\begin{array}{l}\text { Dizygotic } \\
\text { (DZ) }\end{array}$ & $\begin{array}{l}\text { Monozygotic } \\
(M Z)\end{array}$ \\
\hline BWS twins in this stu & $1.00(3 / 0)$ & $0.900(9 / 1)$ \\
\hline Prevalence among normal MZ twins & 0.496 & 0.514 \\
\hline
\end{tabular}

Zygosity, placentation and female-to-male ratio of 13 BWS twins in this study compared with normal twins.

${ }^{a}$ Includes BWS triplet. Data on zygosity and placentation in the normal population are obtained from ${ }^{43}$ data on the normal female-to-male ratio are obtained from. ${ }^{14}$

${ }^{\mathrm{b}}$ Two out three dizygotic twins are conceived by ART, both twins are dichorionic/diamniotic.

of hypomethylation in DNA from the buccal swab and from the blood. The second sib (3B) also showed aberrant methylation in her buccal swab, but at a lower level than in her blood. No loss of methylation could be detected in the third sib (3C). Blood samples of the triplet were taken with a 6-year interval. Methylation levels are comparable between the two samples taken from each twin, and also comparable with DNA from saliva of all three sibs. The tongue of twin $3 \mathrm{~A}$ was surgically reduced, and DNA isolated from this material showed an equal level of LOM compared with lymphocyte DNA.

In the remaining $\mathrm{MZ}$ twin who shared a placenta (twin 7), the percentage of trisomic cells was determined in the blood and fibroblasts of both twins. ${ }^{27}$ In the blood of both twins, 50\% trisomic cells were detected, whereas they were absent in fibroblasts of the non-affected twin.

In all (6 of 6) twins, both $\mathrm{MZ}$ and $\mathrm{DZ}$, with separate placentas, the demethylation of DMR2 was detected only in the blood of the affected child. An exception was twin 10 , in whose case mild demethylation was found in the affected twin, and more severe demethylation was detected in the non-affected twin.
Methylation on loci outside the 11 p15 region was analysed in 10 of 13 twins in this study. In 50\% (5 of 10), hypomethylation of at least one other imprinted locus was detected (4 of $6 \mathrm{MZ}$ MC-DA, 0 of $2 \mathrm{MZ}$ DC-DA and 1 of 2 DZ).

In two cases (twin 2 and twin 11), a modest degree of demethylation of two paternally imprinted genes, H19 and DLK1, was observed. The decreased methylation level of H19 was detected both by HRMA and MS-PCR (see Figure $2 \mathrm{c}$ and $\mathrm{d}$ ). In both twins, the $\mathrm{H} 19$ region analysed by HRMA was sequenced, revealing no SNPs in the fragment.

The levels of demethylation were comparable between the sibs of each twin pair at all loci analysed.

In addition, the methylation levels of DMR2 were also studied in the saliva of twins A, D and J. They showed, as in blood, demethylation of DMR2 in both the affected and non-affected twin.

The level of X-chromosome inactivation was determined in twins $\mathrm{A}, \mathrm{F}$ and $\mathrm{M}$. The inactivation levels of both twins were almost identical (A1: 79\%; A2: 74\%, F1: 73\%, F2: 69\%; M1: 79\%, M2: 64\%) and not skewed.

\section{Phenotype}

The phenotypic data of the BWS twins are summarised in Supplementary Table 3.

All twins, DZ and MZ, were discordant for clinical manifestations typical for BWS. Although in almost all cases the genetic defect is demethylation of DMR2, relative birthweight centiles were calculated for or against twin referents. ${ }^{29}$ Of the three major criteria, macrosomia (birthweight above birth weight centile P9), macroglossia and abdominal wall defects, macrosomia was present only in 5 of the 11 affected twins (twins 6, 7, 11 and 13). In 4 of 10 cases, the unaffected twin had a higher birthweight compared with the affected twin (twins 1, 3B, 9 and 10). Macroglossia was present in 10 of 11 twins, whereas in 9 of 11 cases an abdominal wall defect was present (in most cases, an umbilical hernia). In two twins (twins 5 and 7), the twin-to-twin-transfusion syndrome (TTTS) was observed, with the affected twin as recipient and the unaffected twin as donor.

In many of the twins, the duration of the pregnancy was shorter than the average for normal twins $\left(37\right.$ weeks $\left.^{29}\right)$. In three out of six twins (twins 1, 2 and 3) with complex demethylation, the duration was $<33$ weeks, and the twin with trisomy was also born preterm (30 weeks).

In four MZ MC-DA twins, some signs of BWS were present in the non-affected twin: twin 2B had an abdominal wall defect (umbilical hernia) and hypoglycaemia at birth, twin 3B had macrosomia and mild macroglossia, whereas both twins 3B and 3C had hypoglycaemia, twin 5B also showed hypoglycaemia and twin 6B had an abdominal wall defect (umbilical hernia). 
Table 2 Epigenetic data

\begin{tabular}{|c|c|c|c|c|c|c|c|c|c|c|c|c|}
\hline \multirow[b]{3}{*}{ Twin } & \multicolumn{3}{|c|}{$A^{\prime} d a m^{\mathrm{a}}$} & \multirow{2}{*}{\multicolumn{9}{|c|}{$\begin{array}{l}\text { Salisbury } \\
\text { Blood }\end{array}$}} \\
\hline & \multirow{2}{*}{\multicolumn{2}{|c|}{\begin{tabular}{lc}
\multicolumn{2}{c}{ Blood } \\
DMR1 & DMR2 \\
$($ H19) & $($ KCN1OT1 $)$
\end{tabular}}} & \multirow{2}{*}{$\begin{array}{c}\text { Buccal swab } \\
\text { DMR2 } \\
\text { (KCNQ1OT1) }\end{array}$} & & & & & & & & & \\
\hline & & & & $\begin{array}{l}\text { DMR1 } \\
(H 19)\end{array}$ & \multicolumn{2}{|r|}{$\begin{array}{c}\text { DMR2 } \\
(\text { KCNQ1OT1) }\end{array}$} & $A R H I$ & Grb10 & NESPAS & MEST1 & TNDM & $I G F 2 R$ \\
\hline & & & & & & $M Z, M C-D A$ & & & & & & \\
\hline $1 \mathrm{~A}$ & $w t$ & 0.08 & $\mathrm{~A} 3$ & $w t$ & $w t$ & 10.5 & 1.7 & 9.0 & wt & wt & wt & wt \\
\hline 1B & $w t$ & 0.08 & wt & $w t$ & $w t$ & 8.5 & 1.7 & 9.0 & wt & wt & wt & wt \\
\hline $2 \mathrm{~A}$ & $A 1$ & 0.20 & $\mathrm{~A} 2$ & 1.4 & 3.0 & 2.0 & 1.4 & 3.0 & 4.0 & 3.0 & wt & 5.0 \\
\hline $2 B$ & $A 1$ & 0.21 & wt & 1.4 & 3.0 & 2.0 & 1.4 & 3.0 & 4.0 & 3.0 & wt & 5.0 \\
\hline $3 \mathrm{~A}$ & $w t$ & 0.10 & $\mathrm{~A} 3$ & $w t$ & $w t$ & 3.5 & 2.5 & wt & 2.0 & wt & wt & wt \\
\hline $3 B$ & $w t$ & 0.07 & $\mathrm{~A} 2$ & $w t$ & $w t$ & 5.0 & 2.5 & wt & 2.0 & wt & wt & wt \\
\hline $3 C$ & $w t$ & 0.10 & $N$ & $w t$ & $w t$ & 4.0 & 2.5 & wt & 2.0 & wt & wt & wt \\
\hline $4 \mathrm{~A}$ & $w t$ & 0.12 & $\mathrm{~A} 2$ & $w t$ & $w t$ & 5.0 & wt & wt & wt & wt & wt & wt \\
\hline $4 B$ & $w t$ & 0.19 & wt & $w t$ & $w t$ & 3.5 & wt & wt & wt & wt & wt & wt \\
\hline $5 \mathrm{~A}$ & $w t$ & 0.25 & $\mathrm{~A} 2$ & $w t$ & $w t$ & 2.0 & 2.0 & wt & wt & wt & wt & wt \\
\hline $5 B$ & $w t$ & 0.22 & wt & $w t$ & $w t$ & 2.0 & 2.0 & wt & wt & wt & wt & wt \\
\hline $6 \mathrm{~A}$ & $w t$ & 0.18 & $\mathrm{~A} 2$ & $w t$ & $w t$ & 3.0 & wt & wt & wt & wt & wt & wt \\
\hline $6 B$ & $w t$ & 0.19 & wt & $N D$ & $N D$ & ND & ND & ND & ND & ND & ND & ND \\
\hline $7 A^{c}$ & 0.66 & 0.33 & NA & $N D$ & $N D$ & ND & ND & ND & ND & ND & ND & ND \\
\hline $7 B$ & 0.71 & 0.35 & NA & $N D$ & $N D$ & ND & ND & ND & ND & ND & ND & ND \\
\hline & & & & & & MZ, DC-DA & & & & & & \\
\hline $8 A^{m}$ & $w t$ & 0.20 & $\mathrm{~A} 2$ & $N D$ & $N D$ & ND & ND & ND & ND & ND & ND & ND \\
\hline $8 \mathrm{~B}^{\mathrm{m}}$ & $w t$ & wt & wt & $N D$ & $N D$ & ND & ND & ND & ND & ND & ND & ND \\
\hline $9 \mathrm{~A}$ & $w t$ & 0.12 & NA & $w t$ & $w t$ & 9.5 & wt & wt & wt & wt & wt & wt \\
\hline $9 B$ & $w t$ & wt & NA & $w t$ & $w t$ & wt & wt & wt & wt & wt & wt & wt \\
\hline $10 \mathrm{~A}$ & $w t$ & $\mathrm{~A} 3$ & $\mathrm{~A} 2$ & $w t$ & $w t$ & 1.4 & wt & wt & wt & wt & wt & wt \\
\hline $10 \mathrm{~B}$ & $w t$ & $\mathrm{~A} 2$ & NA & $N D$ & $N D$ & ND & ND & ND & ND & ND & ND & ND \\
\hline & & & & & & DZ & & & & & & \\
\hline $11 \mathrm{~A}$ & $A 1$ & 0.23 & $\mathrm{~A} 2$ & 1.3 & 1.7 & 4.5 & 2.5 & wt & wt & wt & wt & 6.0 \\
\hline $11 \mathrm{~B}$ & $w t$ & wt & wt & $w t$ & $w t$ & wt & wt & wt & wt & wt & wt & wt \\
\hline $12 A^{d}$ & $w t$ & wt & NA & $N D$ & $N D$ & ND & ND & ND & ND & ND & ND & ND \\
\hline $12 \mathrm{~B}$ & $w t$ & wt & NA & $N D$ & $N D$ & ND & ND & ND & ND & ND & ND & ND \\
\hline $13 \mathrm{~A}$ & $w t$ & 0.35 & $\mathrm{~A} 2$ & $N D$ & $w t$ & 1.4 & wt & wt & wt & wt & wt & wt \\
\hline $13 B$ & $w t$ & wt & wt & $N D$ & $w t$ & ND & wt & wt & wt & wt & wt & wt \\
\hline
\end{tabular}

Epigenetic data of 13 BWS twins. Twin A, BWS twin; twin B, non-affected twin; Paternally imprinted genes are indicated in italics, all other loci are maternally imprinted. wt, wild type; ND, not determined; NA, not available; $M$, male twin pair.

${ }^{\mathrm{a}}$ Methylation studies performed by HRMA (results denoted in letters representing a relative level of the defect ${ }^{30}$ ) of southern blot analysis (results denoted in numbers representing the methylation index. ${ }^{9}$ )

${ }^{b}$ Methylation studies performed by MS-PCR (results denoted numerically, where $1=$ normal, hemizygous methylation and numbers $>1$ represent increasing levels of hypomethylation).

${ }^{C}$ Mosaic trisomy 11 p15 detected. ${ }^{27}$

${ }^{\mathrm{d}}$ CDKN1C mutation analysis negative.

None of the non-affected twins fulfilled the clinical criteria for BWS.

\section{Discussion}

This study extends our current understanding of the relationship between $\mathrm{MZ}$ twinning, early developmental processes and epigenetic disorders. We confirm earlier observations that $\mathrm{MZ}$ twinning rates are increased among BWS patients and that BWS MZ twins are predominantly discordant and frequently female. However, our observations present some challenges to current hypotheses about the mechanisms and consequences of DMZ twinning.
Excess of twins and females in BWS patients

In our cohort, we identified 13 twin pairs (12 twin pairs and one triplet) among 400 BWS patients (3\%): three were DZ $(0.8 \%)$, a frequency similar to that in the normal population, whereas 10 were $\mathrm{MZ}$, or $2.3 \%$ compared with $0.3 \%$ in the normal population. The excess of $\mathrm{MZ}$ twins among BWS patients with DMR2 hypomethylation suggests that the methylation defect and the twinning process are correlated. All the MZ BWS twins were diamniotic, suggesting that the shared causative event occurred at approximately days 4-9 of zygotic development.

This study also confirms the excess of females among BWS multiple births, as 12 of the 13 twins described were females. This female excess might be related to X-chromo- 
a

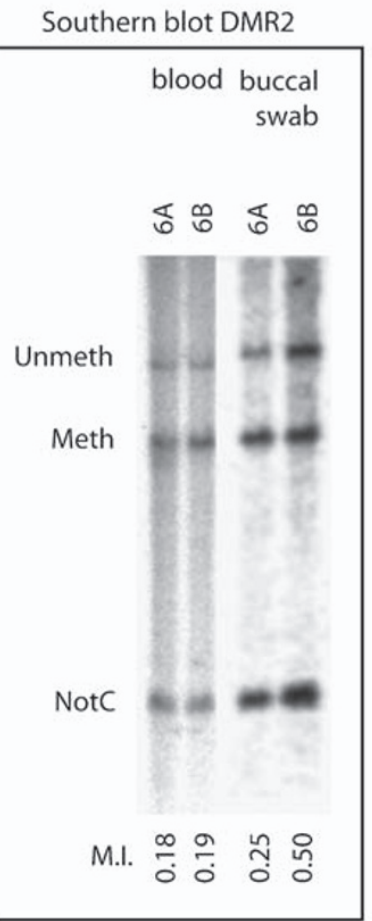

C

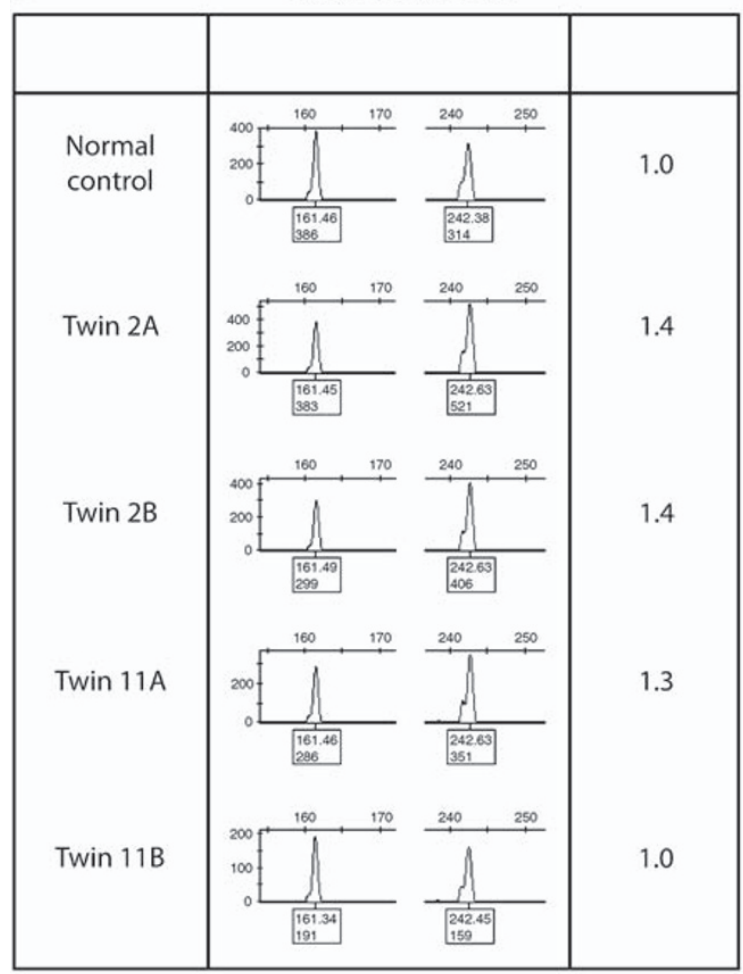

b
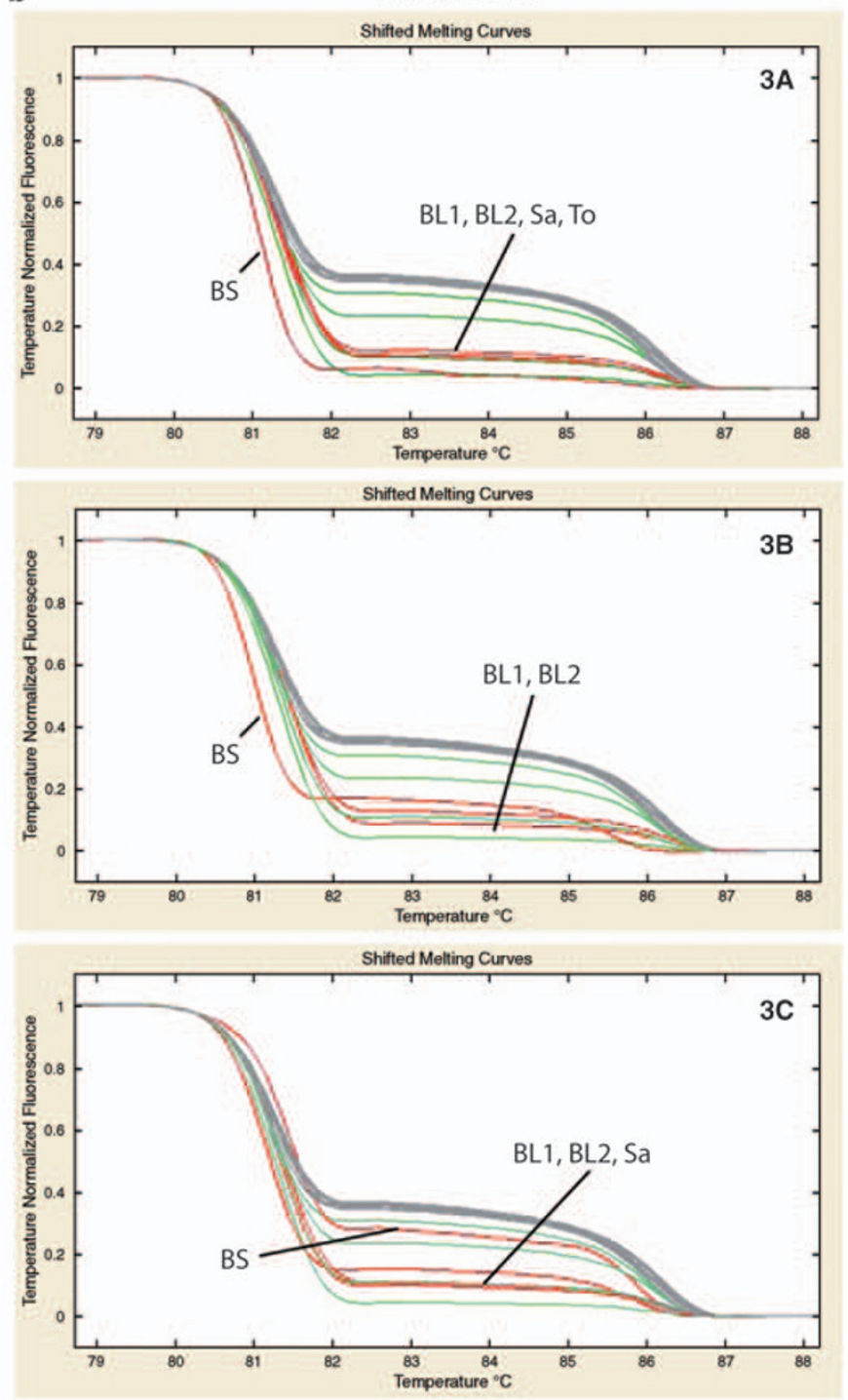

d

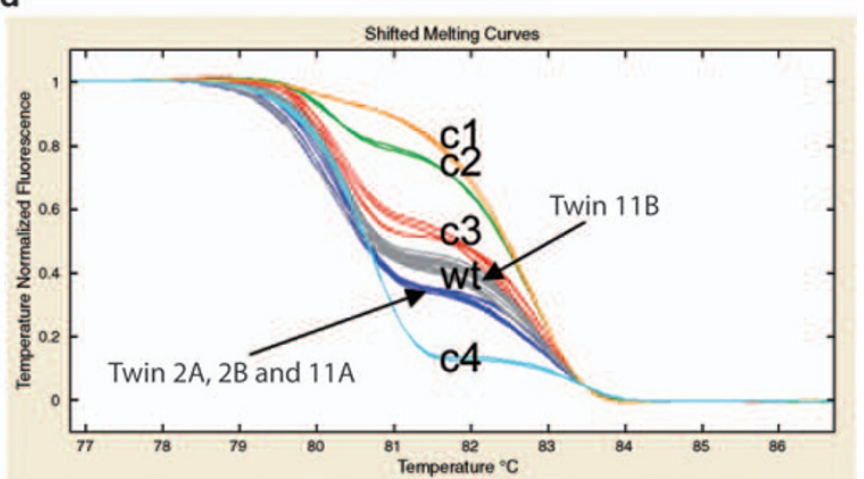

Figure 2 Methylation analysis. (a) Results of methylation analysis of DMR2 of twin 6 performed by southern blot. ${ }^{9}$ Left lanes DNA isolated form blood lymphocytes, right lanes DNA isolated from buccal swabs. NotC: probe to monitor complete digestion. (b) Results of methylation analysis HRMA. ${ }^{30}$ (c) Results methylation analysis of H19 (DRM1) by MS-PCR. ${ }^{13}$ (d) Results methylation analysis of H19 (DRM1) by HRMA of twins 2 and 11. C1-3 control samples with increased methylation levels, C4, control with decreased methylation level, $\mathrm{N}$ normal control. 
some inactivation, twinning, BWS or all of these. Similar to Weksberg et $a l^{24}$ we found no evidence for a specific association between skewed X-chromosome inactivation and BWS among our MZ twins. It is possible that the biological mechanisms of X-chromosome inactivation interfere or interact with the maintenance of methylation at the autosomal imprinted loci. However, as X-chromosome inactivation occurs only in females, and some male MZ twins discordant for BWS have been described, the process of X-chromosome inactivation itself cannot be the only factor affecting the methylation of the BWS locus.

\section{Excess of LOM of DMR2 in BWS twins}

In the majority of twins (11 of 13), a methylation defect of DMR2 could be detected, supporting the theory of Bestor ${ }^{23}$ that a failure of methylation maintenance results in twinning and a methylation defect in the affected twin. This failure is thought to occur at the eight-cell blastocyst stage, preceding the moment that MZ MC DA twinning takes place (days 4-9). However, Bestor's theory ${ }^{23}$ raises the question why not all methylation defects result in twinning, whereas the majority of the BWS cases are singletons. An explanation might be that twinning did occur in all cases, but that the second fetus was resorbed early in pregnancy (vanishing twin, reviewed by Landy and Keith ${ }^{34}$ ).

In one case (twin 12), the genetic cause is unknown, whereas the genetic cause of BWS in twin 7 is trisomy 11p15. The latter case is not a methylation defect as such, but was probably caused by a mitotic non-homologous recombination event. That is also observed by O'Donnell et $a l^{35}$ in a twin discordant for trisomy 21 and by Rohrer et $a l^{36}$ in a twin discordant for the Turner's syndrome.

\section{Excess of HIL in twins}

This study shows for the first time that a subset of discordant MZ twins with BWS manifest hypomethylation of multiple imprinted loci (HIL). This multiple hypomethylation has been described in $\sim 25 \%$ of patients with BWS caused by hypomethylation of DMR2 ${ }^{11}$ Bliek et al, 2008). The HIL in this cohort differs from the earlier studies in two respects: first, it was present in four of the seven twins of whom material was available, and second, it affected not only maternally methylated loci but also paternally methylated loci, H19 and DLK1. It remains to be seen whether it has a different underlying biology from that seen in singleton BWS cases, or whether a genetic cause exists such as the mutation of ZFP57 seen in some patients with transient neonatal diabetes and HIL. ${ }^{37}$

\section{Presence of anomaly in blood and not (generally) in buccal cells of unaffected twin}

Part 1: anomaly present in blood of both twins In all twins with shared placentation in leukocyte DNA, aberrant methylation was found in both the affected twin and the healthy twin. As 70\% of all MZ monochorionic twins share blood through vascular connections, ${ }^{19}$ it is generally postulated that the aberrant methylation found in the blood of the unaffected twin results from fetal circulation sharing through shared blood vessels in the placenta. This exchange includes the exchange of haematopoietic stem cells (HSCs) present in circulating blood. ${ }^{16,35}$

However, for two reasons this theory does not explain the observation that an equivalent and severe hypomethylation was detected in leukocyte DNA of both the affected and unaffected twins.

First, even in a case of complete hypomethylation in an affected twin, circulation sharing would be expected to result in an apparent twofold hypomethylation in the blood of both twins (see Supplementary Figure 3). In this study, the twins 1, 3, 4 and 6 show methylation levels indicating the presence of more than $75 \%$ aberrant lymphocytes in the blood of both the affected and nonaffected twins. This is also observed in other studies. ${ }^{24}$

Second, as the fraction of aberrant cells is about equal in both twins, the HSC pool is expected to be identical. The exchange of the entire HSC population through vascular connections seems unlikely, as the majority of the HSCs home early in development to either the spleen or the liver and in a later stage to the bone marrow (see Supplementary Figure 4). Therefore, the severe hypomethylation sometimes seen in the blood of both twins may derive not merely from circulation sharing, but rather from a common source of HSCs with aberrant methylation.

The first signs of blood cell and vessel formation (angiogenesis and vasculogenesis) are found in the mesoderm of the yolk sac (reviewed by Oostra et $a l^{38}$ ). The yolk sac is an early discernible structure in embryonic development, the anlage of which is formed during the second week of gestation in the human embryo. It faces the lower surface of the embryonic disk, comparable to the amnion, which faces the upper surface. The first phase of gastrulation is characterized by the formation of the extraembryonic mesoderm, which covers both the amnion and the yolk sac membranes. Subsequently, part of the yolk sac epithelium facing the embryonic disc, called hypoblast, is replaced by endoderm. In a later stage when the embryonic disk starts to fold, this part of the yolk sac is incorporated in the embryonic body and will give rise to the intestinal tract and parts of the urogenital system. It is no surprise that the first HSCs appear in the yolk sac mesoderm, as in all non-placental vertebrates the contents of the yolk sac are the only source of nutrients to the developing embryo, the use of which requires a circulatory system.

Up to recently, it was assumed that adult HSCs originate from the embryonic aorta-mesonephros-gonad (AMG) region and that the HSC precursors found in the yolk sac only serve the gastrulating embryo (reviewed by Ueno and Weissman ${ }^{39}$ ). However, Samokhvalov et al ${ }^{40}$ have unequivocally shown that in mice, adult HSCs are indeed derived 
from yolk sac precursors, at least partially. In view of our hypothesis that BWS twins share a common HSC source, one could imagine that the twinning process itself starts after the origin of this source, that is, the extra-embryonic mesoderm covering the yolk sac has formed. Although this seems conceivable only for monoamniotic twinning, the presence of a single yolk sac on ultrasound investigations in monochorionic diamniotic human twin pregnancies (as represented in Figure 3) has been reported to occur to such a degree that it is discouraged to use the number of yolk sacs to determine amnionicity. ${ }^{41}$

This hypothesis, that the affected and non-affected twin share a common yolk sac, does not explain the fact that these aberrant cells account for more than $75 \%$ of all lymphocytes in both the affected and non-affected twin. That can be explained only if the yolk sac was derived from

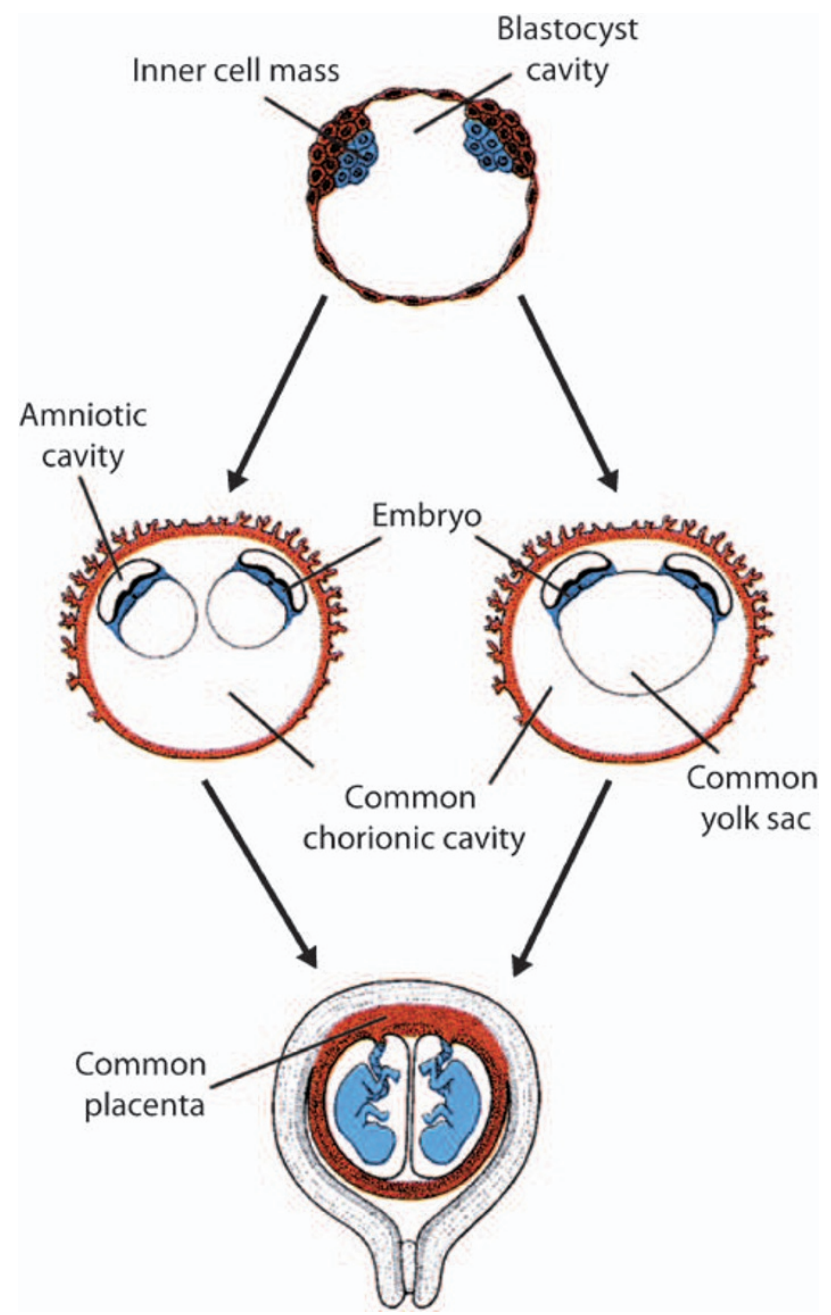

Figure 3 Common yolk-sac (adapted from Langman's Medical Embryology 9th Edition (Sadler 2008)). Two separate mechanisms in yolk-sac formation during the development of monochorionic diamniotic (MC-DA) monozygotic twins. the affected twin. Another option, that HSCs with a methylation defect have a growth advantage over the normal cells and therefore replace the normal cell in the HSC pool, is not likely because methylation studies performed at different time points in BWS patients show stable methylation levels during life (see twin 3 in Figure $2 \mathrm{~b}$ and DJ Mackay, unpublished results). It cannot be excluded that HSCs have a growth advantage only during a certain stage of development and not after birth. It should also be noted that DNA derived from saliva samples primarily contain leukocyte DNA, and therefore, similar to blood-derived DNA, cannot be used to distinguish discordant twins. Genetic testing in these cases should be performed on other tissues such as buccal swabs.

Presence of anomaly in blood and not (generally) in buccal cells of the unaffected twin

Part 2: anomaly not (normally) in buccal cells of unaffected twin We observed that in twins with shared placentation, imprinting anomalies seen in leukocyte DNA of both twins were generally seen in buccal swab DNA of only the unaffected twin. In the triplets that shared a single placenta, LOM of DMR2 was detected in DNA isolated from buccal swabs from the affected (3A) and the mildly affected twin (3B). Triplet 3A had similar levels of LOM in blood and buccal swab DNA, whereas triplet 3B showed milder hypomethylation in buccal swab than in blood. Buccal swab DNA from 3C showed normal methylation.

The presence of a methylation defect in two out of three triplets indicates that it must have arisen before the splitting of the zygote; the unequal methylation defect in the affected triplets suggests an unequal distribution of aberrant cells in the twinning process. This supports the theory of Bestor ${ }^{23}$ that the methylation defect precedes and possibly triggers the twinning event. The BWS features in triplet 3B may result from the small population of methylation-defective cells in this individual.

Imperfect phenotypic discordance: cause is either low level of aberrant cells or altered gene expression transferred through circulation

No twins in our series were concordant for BWS, but in 4 of $10 \mathrm{MZ}$ cases, signs of BWS such as macrosomia, abdominal wall defects, macroglossia and hypoglycaemia were seen in healthy twins. This was also observed in earlier case reports. The unaffected twin of an MZ pair described by Clayton-Smith ${ }^{43}$ showed macroglossia, naevus flammeus and earlobe creases. ${ }^{42,43}$ In another MZ twin pair, ${ }^{21}$ the unaffected twin showed a small umbilical hernia, and transient neonatal macroglossia and naevus flammeus were observed by Olney ${ }^{42}$ in a further unaffected MZ twin. In none of these three cases was the aetiology of disease established. By contrast, $\mathrm{Smith}^{26}$ described a twin pair with DMR1 hypermethylation who were concordant but showed minor phenotypic differences, twin A presenting 
with umbilical hernia and twin B with naevus flammeus. The BWS features present in less-affected twins may, similar to those in the triplets $3 \mathrm{~A}-3 \mathrm{C}$, be explained by a low level of mosaicism in certain tissues of these individuals. Possibly, the number of aberrant cells in the buccal swabs of these individuals does not reach the detection levels of the methylation test. It is also possible that less-affected twins do not harbour cells with methylation defects, except in their circulation. Their BWS features may be caused by altered expression in the circulation of genes controlled by DMR2.

In conclusion, we have confirmed the prevalence of $\mathrm{MZ}$ twins with discordant presentation and of female twins among BWS patients. We have shown that 5 of 10 discordant MZ BWS twins have a generalized hypomethylation of imprinted genes - including, uniquely, paternally methylated loci - throughout the genome. We show that methylation patterns and phenotypes of a BWS triplet support the theory that methylation failure precedes and possibly triggers the twinning process. We propose a mechanism, involving migration of the blood cell precursor cells from the yolk sac to both twins, to explain similar aberrant methylation found in blood cells of discordant twin pairs.

\section{Acknowledgements}

We thank $S$ Kant, D Marcus-Soekarman and $L$ Yates for the assessment of patients and collection of clinical data, and Professor RMC Hennekam for helpful discussions. We thank $E$ Roos for the layout of the figures in this paper, and we also thank all the twins and their families for participating in this study.

\section{References}

1 Beckwith JB: Extreme Cytomegaly of the Adrenal Fetal Cortex, Omphalocele, Hyperplasia of Kidneys and Pancreas, and Leydig-Cell Hyperplasia: Another Syndrome? Los Angeles: Western Society for Pediatric Research, 1963.

2 Wiedemann HR: Familial malformation complex with umbilical hernia and macroglossia: a new syndrome? J Genet Hum 1964; 13: 223-232.

3 Wiedemann HR: Tumours and hemihypertrophy associated with Wiedemann Beckwith syndrome. Eur J Pediatr 1983; 141: 129.

4 Sotelo-Avila C, Gonzalez-Crussi F, Fowler JW: Complete and incomplete forms of Beckwith-Wiedemann syndrome: their oncogenic potential. J Pediatr 1980; 96: 47-50.

5 Pettenati MJ, Haines JL, Higgins RR et al: Wiedemann-Beckwith syndrome: presentation of clinical and cytogenetic data on 22 new cases and review of the literature. Hum Genet 1986; 74: $143-154$.

6 Debaun MR, Tucker MA: Risk of cancer during the first four years of life in children from The Beckwith-Wiedemann Syndrome Registry. J Pediatr 1998; 132: 398-400.

7 Weksberg R, Shuman C, Smith AC: Beckwith-Wiedemann syndrome. Am J Med Genet C Semin Med Genet 2005; 137C: 12-23.

8 Cooper WN, Luharia A, Evans GA et al: Molecular subtypes and phenotypic expression of Beckwith-Wiedemann syndrome. Eur I Hum Genet 2005; 13: 1025-1032.
9 Bliek J, Maas SM, Ruijter JM et al: Increased tumour risk for BWS patients correlates with aberrant H19 and not KCNQ1OT1 methylation: occurrence of KCNQ1OT1 hypomethylation in familial cases of BWS. Hum Mol Genet 2001; 10: 467-476.

10 Reik W, Maher ER: Imprinting in clusters: lessons from Beckwith-Wiedemann syndrome. Trends Genet 1997; 13: 330-334.

11 Rossignol S, Steunou V, Chalas C et al: The epigenetic imprinting defect of patients with Beckwith-Wiedemann syndrome born after assisted reproductive technology is not restricted to the $11 \mathrm{p} 15$ region. J Med Genet 2006; 43: 902-907.

12 Bliek J, Verde G, Callaway JL et al: Hypomethylation at multiple maternally methylated imprinted regions including PLAGL1 and GNAS loci in Beckwith-Wiedemann syndrome. Eur J Hum Genet 2009; 17: 611-619.

13 Mackay DJ, Hahnemann JM, Boonen SE et al: Epimutation of the TNDM locus and the Beckwith-Wiedemann syndrome centromeric locus in individuals with transient neonatal diabetes mellitus. Hum Genet 2006; 119: 179-184.

14 James WH: Sex ratio and placentation in twins. Ann Hum Biol 1980; 7: 273-276.

15 Kondo S, Schutte BC, Richardson RJ et al: Mutations in IRF6 cause Van der Woude and popliteal pterygium syndromes. Nat Genet 2002; 32: 285-289.

16 Verona LL, Damian NG, Pavarina LP et al: Monozygotic twins discordant for Goldenhar syndrome. J Pediatr (Rio J) 2006; 82: $75-78$.

17 Robertson SP, Thompson S, Morgan T et al: Postzygotic mutation and germline mosaicism in the otopalatodigital syndrome spectrum disorders. Eur J Hum Genet 2006; 14: 549-554.

18 De GL, Jinnah HA, Harris JC et al: Lesch-Nyhan disease in a female with a clinically normal monozygotic twin. Mol Genet Metab 2005; 85: 70-77.

19 Hall JG: Twins and twinning. Am J Med Genet 1996; 61: 202-204.

20 Walker SP, Meagher S, White SM: Confined blood chimerism in monochorionic dizygous (MCDZ) twins. Prenat Diagn 2007; 27: 369-372.

21 Orstavik RE, Tommerup N, Eiklid $\mathrm{K}$ et al: Non-random $\mathrm{X}$ chromosome inactivation in an affected twin in a monozygotic twin pair discordant for Wiedemann-Beckwith syndrome. Am J Med Genet 1995; 56: 210-214.

22 Machin GA: Some causes of genotypic and phenotypic discordance in monozygotic twin pairs. Am J Med Genet 1996; 61: 216-228.

23 Bestor TH: Imprinting errors and developmental asymmetry. Philos Trans $R$ Soc Lond B Biol Sci 2003; 358: 1411-1415.

24 Weksberg R, Shuman C, Caluseriu O et al: Discordant KCNQ1OT1 imprinting in sets of monozygotic twins discordant for Beckwith-Wiedemann syndrome. Hum Mol Genet 2002; 11: $1317-1325$

25 Li M, Squire J, Shuman C et al: Imprinting status of 11p15 genes in Beckwith-Wiedemann syndrome patients with CDKN1C mutations. Genomics 2001; 74: 370-376.

26 Smith AC, Rubin T, Shuman C et al: New chromosome 11p15 epigenotypes identified in male monozygotic twins with Beckwith-Wiedemann syndrome. Cytogenet Genome Res 2006; 113: $313-317$.

27 Marcus-Soekarman D, Hamers G, Velzeboer S et al: Mosaic trisomy $11 \mathrm{p}$ in monozygotic twins with discordant clinical phenotypes. Am J Med Genet A 2004; 124A: 288-291.

28 Gaston V, Le BY, Soupre V et al: Analysis of the methylation status of the KCNQ1OT and H19 genes in leukocyte DNA for the diagnosis and prognosis of Beckwith-Wiedemann syndrome. Eur J Hum Genet 2001; 9: 409-418.

29 Gielen M, Lindsey PJ, Derom C et al: Twin-specific intrauterine 'growth' charts based on cross-sectional birthweight data. Twin Res Hum Genet 2008; 11: 224-235.

30 Alders $\mathrm{M}$, Bliek J, van der Lip $\mathrm{K}$ et al: Determination of KCNQ1OT1 and H19 methylation levels in BWS and SRS patients 
using high resolution melting analysis. Hum Mutat 2009; 17: 467-473.

31 Allen RC, Zoghbi HY, Moseley AB et al: Methylation of HpaII and HhaI sites near the polymorphic CAG repeat in the human androgen-receptor gene correlates with $\mathrm{X}$ chromosome inactivation. Am J Hum Genet 1992; 51: 1229-1239.

32 Horsthemke B, Nazlican H, Husing J et al: Somatic mosaicism for maternal uniparental disomy 15 in a girl with Prader-Willi syndrome: confirmation by cell cloning and identification of candidate downstream genes. Hum Mol Genet 2003; 12: $2723-2732$.

33 Hall JG: Twinning. Lancet 2003; 362: $735-743$.

34 Landy HJ, Keith LG: The vanishing twin: a review. Hum Reprod Update 1998; 4: 177-183.

35 O'Donnell CP, Pertile MD, Sheffield LJ et al: Monozygotic twins with discordant karyotypes: a case report. J Pediatr 2004; 145: 406-408.

36 Rohrer TR, Gassmann KF, Rauch A et al: Growth of heterokaryotic monozygotic twins discordant for Ullrich-Turner syndrome during the first years of life. Am J Med Genet A 2004; 126A: 78-83.

37 Mackay DJ, Callaway JL, Marks SM et al: Hypomethylation of multiple imprinted loci in individuals with transient neonatal diabetes is associated with mutations in ZFP57. Nat Genet 2008; 40: 949-951.

38 Oostra RJ, Steding G, Lamers WH et al: Steding's and Virágh's Scanning Electron Microscopy Atlas of the Developing Human Heart New York: Springer, 2007

39 Ueno H, Weissman IL: Stem cells: blood lines from embryo to adult. Nature 2007; 446: 996-997.

40 Samokhvalov IM, Samokhvalova NI, Nishikawa S: Cell tracing shows the contribution of the yolk sac to adult haematopoiesis. Nature 2007; 446: 1056-1061.

41 Shen O, Samueloff A, Beller U et al: Number of yolk sacs does not predict amnionicity in early first-trimester monochorionic multiple gestations. Ultrasound Obstet Gynecol 2006; 27: $53-55$.

42 Olney AH, Buehler BA, Waziri M: Wiedemann-Beckwith syndrome in apparently discordant monozygotic twins. Am J Med Genet 1988; 29: 491-499.

43 Clayton-Smith J, Read AP, Donnai D: Monozygotic twinning and Wiedemann-Beckwith syndrome. Am J Med Genet 1992; 42: 633-637.

44 Hall JG: Twinning: mechanisms and genetic implications. Curr Opin Genet Dev 1996; 6: 343-347.

Supplementary Information accompanies the paper on European Journal of Human Genetics website (http://www.nature.com/ejhg) 